\title{
Evaluation of Posterior Astigmatism Measured With Scheimpflug Imaging
}

\author{
Gabor Nemeth, MD, PhD, * Andras Berta, MD, PhD, DSc, * Agnes Lipecz, MD, * Ziad Hassan, MD, PhD, $\uparrow$ \\ Eszter Szalai, MD, PhD,* and Laszlo Modis $J r, M D, P h D, D S c^{*}$
}

Purpose: Our aim was to assess the corneal power, axis, and age dependence of the anterior and posterior corneal surfaces with Scheimpflug imaging.

Methods: Patients older than 10 years without a history of ocular surgery, corneal diseases, contact lens wearing, or severe dry eye were enrolled. Data regarding the anterior and posterior cornea were analyzed by means of Scheimpflug imaging (Pentacam HR).

Results: The median age was 46.8 years (range: 10.0-90.3), and involved 827 eyes of 827 patients. Posterior corneal astigmatism was a median of -0.30 diopters (D) and exceeded $0.50 \mathrm{D}$ in $12.56 \%$. The ratio of the anterior-to-posterior corneal radius was a median of 1.217 (range: 1.021-1.402). The correlation between this ratio and age was significant $(r=-0.219 ; P<0.001)$. The correlation between anterior and posterior corneal astigmatism and age was significant. At the anterior surface, the ratio of with-the-rule astigmatism was $80.0 \%$ for the age range 10 to 20 , decreasing to $36.0 \%$ for those aged over 81 years, whereas the ratio of against-the-rule astigmatism increased from $7.1 \%$ to $44.0 \%$. At the posterior surface, the ratio of with-the-rule astigmatism was $94.3 \%$ for the age range 10 to 20 years, decreasing to $84.0 \%$ for those aged over 81 years, whereas the ratio of against-the-rule astigmatism increased from $1.4 \%$ to $8.0 \%$.

Conclusions: The posterior cornea significantly influences corneal astigmatism. Its power value and orientation are much more stable with advancing age compared with anterior surface values.

Key Words: age dependence, astigmatism, posterior cornea, Scheimpflug imaging

(Cornea 2014;33:1214-1218)

T wo thirds of the refractive power of the human eye is related to the cornea, from which a considerable, negative amount is presented at the posterior surface. The magnitude and orientation of astigmatism are continuously changing from birth onward. ${ }^{1-3}$ Few studies comprising a large number

Received for publication March 24, 2014; revision received July 1, 2014; accepted July 5, 2014. Published online ahead of print August 27, 2014.

From the *Department of Ophthalmology, University of Debrecen, Debrecen, Hungary; and $\dagger$ Orbi-Dent Refractive Surgery and Medical Center, Debrecen, Hungary.

The authors have no funding or conflicts of interest to disclose.

Reprints: Gabor Nemeth, MD, PhD, Department of Ophthalmology, University of Debrecen, Nagyerdei Boulevard 98, H-4012 Debrecen, Hungary (e-mail: nemeth222@yahoo.com).

Copyright (C) 2014 by Lippincott Williams \& Wilkins of patients have been published on astigmatic changes in adults, though they do not emphasize the posterior component of the corneal astigmatism, because of the impossibility of its measurement by common methods. ${ }^{4-9}$ It is possible to measure the radius of the posterior corneal surface using the first and second Purkinje images. ${ }^{10}$ Posterior corneal imaging can be carried out with an OrbScan (Bausch and Lomb, Rochester, NY), a Pentacam (Oculus, Wetzlar, Germany), and its higher resolution form, and with newer combined technology, such as Scheimpflug imaging with a Placido disc (Sirius; Costruzione Strumenti Oftalmici, Florence, Italy) or a Placido disc in combination with a dual-channel Scheimpflug camera using ray tracing (Galilei dual Scheimpflug analyzer; Zeimer Group, Port, Switzerland).

The control of corneal astigmatism during cataract surgery has been of increasing importance. More than $30 \%$ of patients require astigmatism correction during cataract surgery because of the prevalence of corneal astigmatism of $>1.0 \mathrm{D} .^{5,6,9}$

Our aim was to examine the magnitude, orientation, and age-related changes of anterior and posterior corneal astigmatism and to compare their relationships with one another, and to assess the age dependence of their changes in the corneas of healthy patients measured with a high-resolution version of Pentacam.

\section{PATIENTS AND METHODS}

Healthy volunteers aged 10 years or above were chosen for this data collection from among those who came for a routine ophthalmological examination to the Department of Ophthalmology, University of Debrecen. The inclusion criterion was a clinically healthy anterior segment of the eye examined by means of a slit-lamp biomicroscope; the exclusion criteria were any previous ocular surgery, any corneal diseases, a history of contact lens wearing, and severe dry eye, any of which could influence the measurements. If these criteria were realized, routine examinations were completed by taking a picture with the Pentacam HR. One eye was randomly selected from each of these subjects, if both eyes met the inclusion criteria.

The orientation of corneal astigmatism was defined as against-the-rule (ATR) astigmatism in the case of the steepest corneal meridian between 0 to $30^{\circ}$ and 150 to $180^{\circ}$, oblique astigmatism between 30 to $60^{\circ}$ and 120 to $150^{\circ}$, and withthe-rule (WTR) astigmatism between 60 and $120^{\circ}$.

Images were captured with the high-resolution version of Pentacam (Pentacam HR, software version 1.17r139). We 
used Scheimpflug imaging in the 25 images per 2-second mode, and the images were taken in the automatic mode. The keratometry data were recorded at the central 3-mm zone. In the case of image distortion (eg, blinking) or a lack of any observed data, the image taken was repeated.

The Pentacam HR uses a 475-nm monochromatic blue light for imaging, whereas the camera takes images at a resolution of $1392 \times 1040$ pixels and uses 138,000 true elevation data points over the whole cornea. The software allows for the automatic analysis of the anterior segment, the anterior and posterior topography of the cornea, pachymetry, and the analysis of the anterior chamber and the lens. Finally, the device creates a 3-dimensional model of the anterior segment. The software of the device is capable of correcting distortions in the Scheimpflug images based on the geometry of the Scheimpflug principle.

The research protocol adhered to the tenets of the Declaration of Helsinki and was approved by the Institutional Ethics Committee. The data preparation and statistical analysis were performed with the Microsoft Excel and MedCalc 10.0 software packages. The normality of the data was tested using the Kolmogorov-Smirnov test. Because the normality was rejected $(P<0.001)$ in all of our examined data, nonparametric tests were used. The descriptive statistical results were presented as median, ranges and interquartile ranges (IQR), and 95\% confidence interval (CI) for the median. The Spearman rank test was applied to explore the correlations. A $P<0.05$ was considered to be statistically significant.

\section{RESULTS}

Our study included 827 randomly selected eyes of 827 patients (498 women and 329 men). The median age of our patients was 46.8 (range: 10-90, IQR: $28.7-67.0,95 \%$ CI for the median: 44.5-49.9) years. It was 48.13 years in females (range: 10.77-90.33 years, IQR: 28.1-67.9 years, 95\% CI for the median: 44.5-54.0 years) and 45.9 years in males (range: 10.0-84.9 years, IQR: $30.6-64.6$ years, $95 \%$ CI for the median: 41.5-49.3 years). There was no statistically significant difference regarding ages between genders $(P=0.48)$. The radius data, keratometric data, and astigmatism data are presented in detail in Table 1 and separated by gender in Table 2. Posterior corneal astigmatism was a median of -0.3 diopters (D) and exceeded $0.5 \mathrm{D}$ in $12.56 \%, 0.75 \mathrm{D}$ in $5.31 \%$, and $1.0 \mathrm{D}$ in $2.41 \%$ of the examined eyes.

The ratio of the anterior-to-posterior corneal radius was a median of 1.217 (range: 1.021-1.402, IQR: 1.200-1.234, 95\% CI for the median: 1.215-1.219). The correlation between this ratio and age was a Spearman correlation of rho $(r)=-0.219(P<0.001)$. The correlation between the magnitude of the anterior and posterior keratometric data at the flattest and steepest corneal meridians was $r=-0.851$ $(P<0.001)$ and $r=-0.815(P<0.001)$. The correlation between the mean anterior and posterior radii of the cornea was $r=0.85(P<0.001)$.

The orientation of the anterior corneal astigmatism was WTR in $65.78 \%$, ATR in $17.77 \%$, and oblique in $16.44 \%$. Regarding the posterior astigmatism, it was WTR in $88.75 \%$, ATR in $3.14 \%$, and oblique in $8.10 \%$. Anterior and posterior astigmatism both have the same axis in the same patient in $67.71 \%$ and were different in $32.29 \%$. The range of the difference between the anterior and posterior axes was -175.9 to $174.8^{\circ}$. It was within $10^{\circ}$ in 385 eyes $(46.55 \%)$ and within $33^{\circ}$ in 620 eyes $(74.97 \%)$. The correlation between anterior, posterior corneal astigmatism, anterior horizontal radius, posterior vertical radius value, and age was significant (Table 3 ).

At the anterior surface, the ratio of WTR astigmatism was $80.0 \%$ for the age range 10 to 20 years, and it decreased rapidly to $36.0 \%$ for ages over 81 years, whereas the ratio of ATR astigmatism increased from $7.1 \%$ to $44.0 \%$. The ratio of the oblique astigmatism was between $12.9 \%$ and $23.5 \%$ (Fig. 1). At the posterior surface, the ratio of WTR astigmatism was $94.3 \%$ for the age range 10 to 20 years, and it decreased to $84.0 \%$ for ages over 81 years, whereas the ratio of ATR astigmatism increased from $1.4 \%$ to $8.0 \%$ and the ratio of oblique astigmatism was nearly the same (Fig. 2).

\section{DISCUSSION}

Corneal astigmatism has both anterior and posterior surface components. Total corneal power is determined by anterior and posterior corneal curvature, corneal thickness, and the difference of refractive indices between air and

TABLE 1. Descriptive Statistical Results From the Anterior and Posterior Surface of the Cornea Obtained by a Pentacam HR

\begin{tabular}{|c|c|c|c|c|c|}
\hline & Median & Minimum & Maximum & IQR & 95\% CI (Median) \\
\hline $\mathrm{Rh} F(\mathrm{~mm})$ & 7.77 & 6.22 & 9.49 & 7.56 to 8.02 & 7.75 to 7.80 \\
\hline $\mathrm{Rv} F(\mathrm{~mm})$ & 7.67 & 5.86 & 9.51 & 7.47 to 7.88 & 7.63 to 7.69 \\
\hline $\mathrm{Rh} \mathrm{B}(\mathrm{mm})$ & 6.49 & 4.64 & 9.64 & 6.32 to 6.70 & 6.47 to 6.53 \\
\hline Rv B (mm) & 6.21 & 4.24 & 8.73 & 6.04 to 6.38 & 6.18 to 6.23 \\
\hline K1 F (D) & 43.2 & 35.5 & 53.6 & 42.00 to 44.30 & 43.10 to 43.30 \\
\hline K2 F (D) & 44.3 & 38.9 & 57.6 & 43.10 to 45.30 & 44.10 to 44.40 \\
\hline K1 B (D) & -6.10 & -8.60 & -4.2 & -6.30 to -6.00 & -6.20 to -6.10 \\
\hline K2 B (D) & -6.50 & -9.4 & -4.5 & -6.70 to -6.30 & -6.50 to -6.40 \\
\hline Astigmatism F (D) & 0.90 & 0.00 & 8.8 & 0.60 to 1.40 & 0.80 to 0.90 \\
\hline Astigmatism B (D) & -0.30 & 0.00 & -1.6 & -0.20 to -0.40 & -0.30 to -0.31 \\
\hline
\end{tabular}

$\mathrm{B}$, back; F, front; K1 B, flattest keratometric reading at the posterior surface; K1 F, flattest keratometric reading at the anterior surface; K2 B, steepest keratometric reading at the posterior surface; $\mathrm{K} 2 \mathrm{~F}$, steepest keratometric reading at the anterior surface; $\mathrm{Rh} \mathrm{B}$, horizontal radius of the cornea at the posterior surface; Rh $\mathrm{F}$, horizontal radius of the cornea at the anterior surface; Rv B, vertical radius of the cornea at the posterior surface; Rv F, vertical radius of the cornea at the anterior surface. 
TABLE 2. Descriptive Statistical Results From the 2 Surfaces of the Cornea Separated by Gender [498 Women (60.22\%) and 329 Men (39.78\%)]

\begin{tabular}{|c|c|c|c|c|c|}
\hline & \multicolumn{2}{|c|}{ Female } & \multicolumn{2}{|c|}{ Male } & \multirow[b]{2}{*}{$P$} \\
\hline & Median & 95\% CI (Median) & Median & 95\% CI (Median) & \\
\hline $\mathrm{Rh} F(\mathrm{~mm})$ & 7.74 & 7.71 to 7.77 & 7.83 & 7.80 to 7.88 & $<0.001 *$ \\
\hline $\mathrm{Rv} F(\mathrm{~mm})$ & 7.60 & 7.58 to 7.65 & 7.74 & 7.69 to 7.78 & $<0.001 *$ \\
\hline $\mathrm{Rh} B(\mathrm{~mm})$ & 6.45 & 6.43 to 6.48 & 6.58 & 6.53 to 6.62 & $<0.001 *$ \\
\hline $\mathrm{Rv} B(\mathrm{~mm})$ & 6.15 & 6.12 to 6.18 & 6.26 & 6.21 to 6.31 & $<0.001 *$ \\
\hline K1 F (D) & 43.45 & 43.26 to 43.60 & 42.80 & 42.60 to 43.00 & $<0.001^{*}$ \\
\hline K2 B (D) & -6.50 & -6.48 to -6.52 & -6.40 & -6.50 to -6.40 & $<0.001 *$ \\
\hline Astigmatism F (D) & 0.90 & 0.90 to 1.00 & 0.80 & 0.70 to 0.90 & 0.83 \\
\hline Astigmatism B (D) & -0.30 & -0.30 to -0.31 & -0.30 & -0.30 to -0.31 & 0.58 \\
\hline
\end{tabular}

B, back; F, front; K1 B, flattest keratometric reading at the posterior surface; K1 F, flattest keratometric reading at the anterior surface; K2 B, steepest keratometric reading at the posterior surface; K2 F, steepest keratometric reading at the anterior surface; Rh B, horizontal radius of the cornea at the posterior surface; Rh F, horizontal radius of the cornea at the anterior surface; Rv B, vertical radius of the cornea at the posterior surface; Rv F, vertical radius of the cornea at the anterior surface.

* Statistical significance of the difference.

anterior corneal surface and between posterior corneal surface and aqueous humor. Previously, the exact measurement of the data of the posterior corneal surface was not possible; thus, the total corneal power was calculated from the anterior surface measurements using modified keratometric indices. The Pentacam calculates the power of the anterior corneal surface using the difference between the refractive index of air $(n=1.0)$ and the refractive index of cornea $(n=1.376)$. The power of the posterior surface is calculated using the difference between the refractive index of cornea and the refractive index of aqueous humor $(\mathrm{n}=1.336)$. Since posterior corneal imaging became possible, it has received increasing clinical interest. It was recognized that the posterior corneal curvature, the elevation data, and the anteroposterior (AP) corneal power ratio are affected just as in the early stages of keratoconus. ${ }^{1-13}$ Some authors have suggested that neglecting the surgically induced astigmatism of the posterior cornea may have a negative impact on surgical procedures for correcting astigmatism ${ }^{14,15}$ Moreover, Bregnhøj and $\mathrm{Næser}^{16}$ have indicated that ocular residual astigmatism largely represents posterior corneal astigmatism in the pseudophakic eye. The posterior cornea causes a net ATR

TABLE 3. Correlations Between the Anterior and Posterior Corneal Parameters and the Age of the Patients

\begin{tabular}{lrc}
\hline & $\boldsymbol{r}$ & $\boldsymbol{P}$ \\
\hline Anterior astigmatism & -0.170 & $<0.001^{*}$ \\
Posterior astigmatism & -0.116 & $<0.001^{*}$ \\
Anterior K1 & 0.057 & 0.099 \\
Posterior K1 & 0.048 & 0.171 \\
Anterior K2 & -0.001 & 0.975 \\
Posterior K2 & 0.086 & $0.013^{*}$ \\
Anterior Rh & -0.109 & $0.001^{*}$ \\
Posterior Rh & 0.029 & 0.412 \\
Anterior Rv & 0.062 & 0.075 \\
Posterior Rv & 0.096 & $0.005^{*}$ \\
\hline
\end{tabular}

*Statistical significance of the correlation. astigmatism to the total cornea if its magnitude is large enough and its meridian corresponds to the meridian of the anterior astigmatism. If we take the posterior corneal astigmatism into consideration, the total corneal astigmatism is less in patients with WTR and more in those with ATR. Therefore, patients with WTR astigmatism will be overcorrected, whereas patients with ATR astigmatism will be undercorrected for toric intraocular lenses (IOL), if we neglect the effect of the posterior cornea, as was written in a study about eyes after toric IOL implantation. ${ }^{17}$ Moreover, we found a strong correlation between the magnitude of the anterior and posterior keratometric data at the flattest and steepest corneal meridians, so the role of the posterior cornea may be magnified in cases when corneas have higher keratometric data.

According to the Gullstrand eye model, the anterior corneal radius is a mean of $7.8 \mathrm{~mm}$, and the posterior radius is a mean of $6.5 \mathrm{~mm}$. The estimated radius of the central posterior corneal curvature is demonstrated as being $6.71 \mathrm{~mm} .{ }^{18}$ Using the Purkinje image method, the mean radius of curvature was $6.42 \mathrm{~mm} .{ }^{19}$ Módis et $\mathrm{al}^{20}$ measured larger mean posterior astigmatism $(-0.78 \mathrm{D})$ with the OrbScan device. Dubbelman et $\mathrm{al}^{21}$ measured $7.79 \mathrm{~mm}$ for the anterior corneal surface radius and $6.53 \mathrm{~mm}$ for the posterior corneal surface with Scheimpflug photography. Ho et $\mathrm{al}^{22}$ found the central radius of curvature of the posterior corneal surface to be $6.34 \mathrm{~mm}$ with the Pentacam, whereas Pinero et $\mathrm{al}^{23}$ obtained $6.47 \mathrm{~mm}$ for the same with the Pentacam. In our measurement obtained by using the Pentacam HR, the median of the anterior radius of the corneal curvature was $7.77 \mathrm{~mm}$ horizontally and $7.67 \mathrm{~mm}$ vertically; the radius of the posterior corneal curvature was $6.49 \mathrm{~mm}$ horizontally and $6.21 \mathrm{~mm}$ vertically. One possible reason for different keratometric data in the literature is the percentage of females in the measured population because there are definite gender differences. ${ }^{7}$ Male eyes had significantly larger corneal radii (flatter keratometry readings) at both surfaces in our study, but we did not show statistically significant differences regarding the front or back surface corneal astigmatism between genders. 


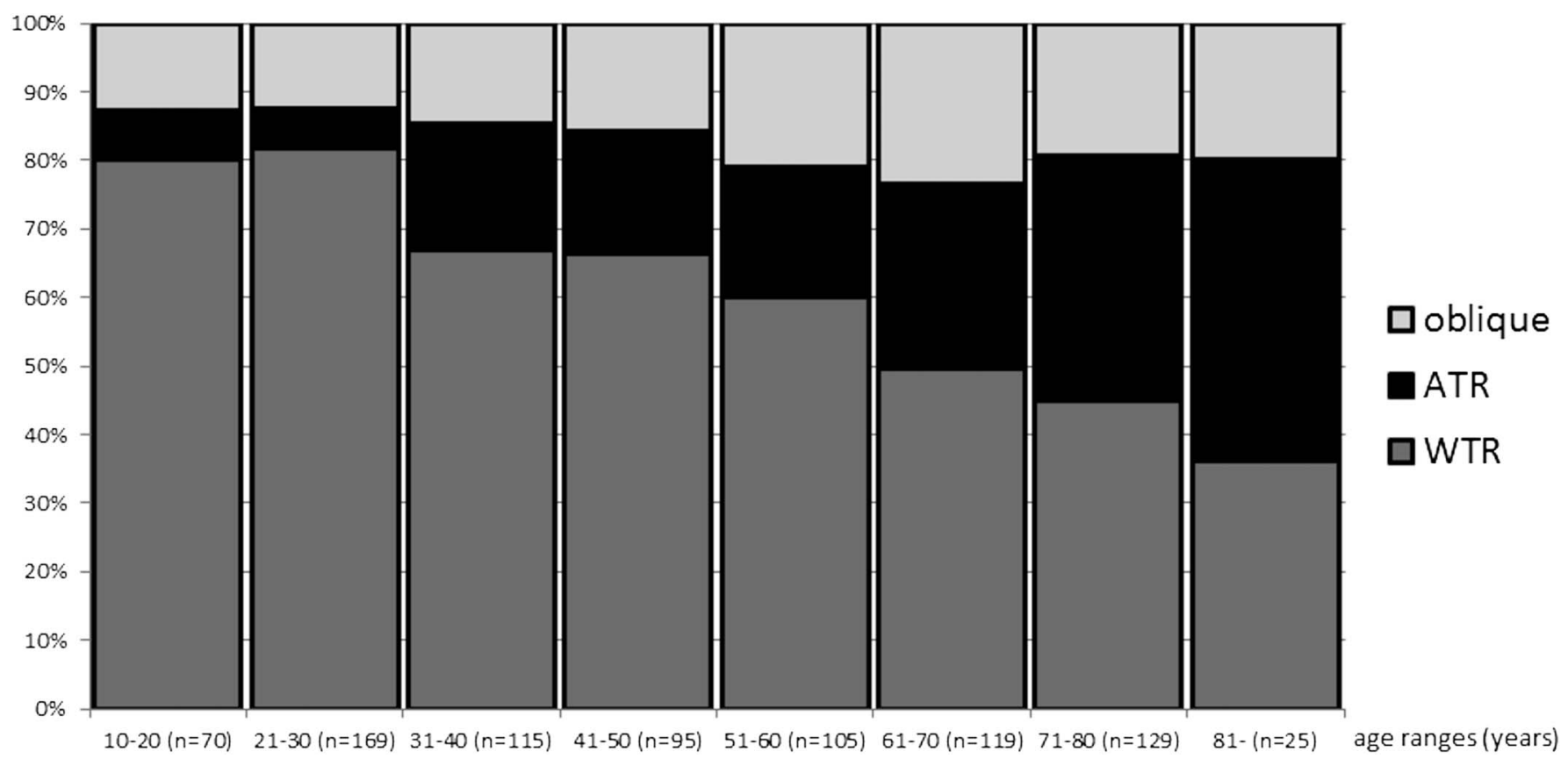

FIGURE 1. Age dependence of the orientation of the anterior corneal astigmatism. The $x$ axis denotes the age groups in years, and the $y$ axis represents the prevalence of types of astigmatism.

In the literature, the ratio of the radius of the anterior corneal curvature to the radius of the posterior corneal curvature (the AP ratio) ranged from 1.132 (the Gullstrand schematic eye model) to 1.235 in the data from Dubbelman et al. $^{24}$ With the Pentacam, a normal distribution was observed for most of the corneal parameters, and 1.223 was measured at the central $4.0-\mathrm{mm}$ corneal zone for the AP ratio. $^{22}$ Monatalbán et $\mathrm{al}^{25}$ reported an AP ratio of 1.19 with the Pentacam, which is smaller than that for our data (1.217). They showed that a significant, positive correlation existed between the AP shape factor (defined as 1+asphericity) ratio and age, as between the curvatures of the anterior, posterior corneal surfaces and age at 4.5- and 8.0-mm corneal diameters. Our data were measured in the $3.0-\mathrm{mm}$ corneal zone, and we observed a negative, statistically significant, but weak relationship between the AP ratio and subject's age.

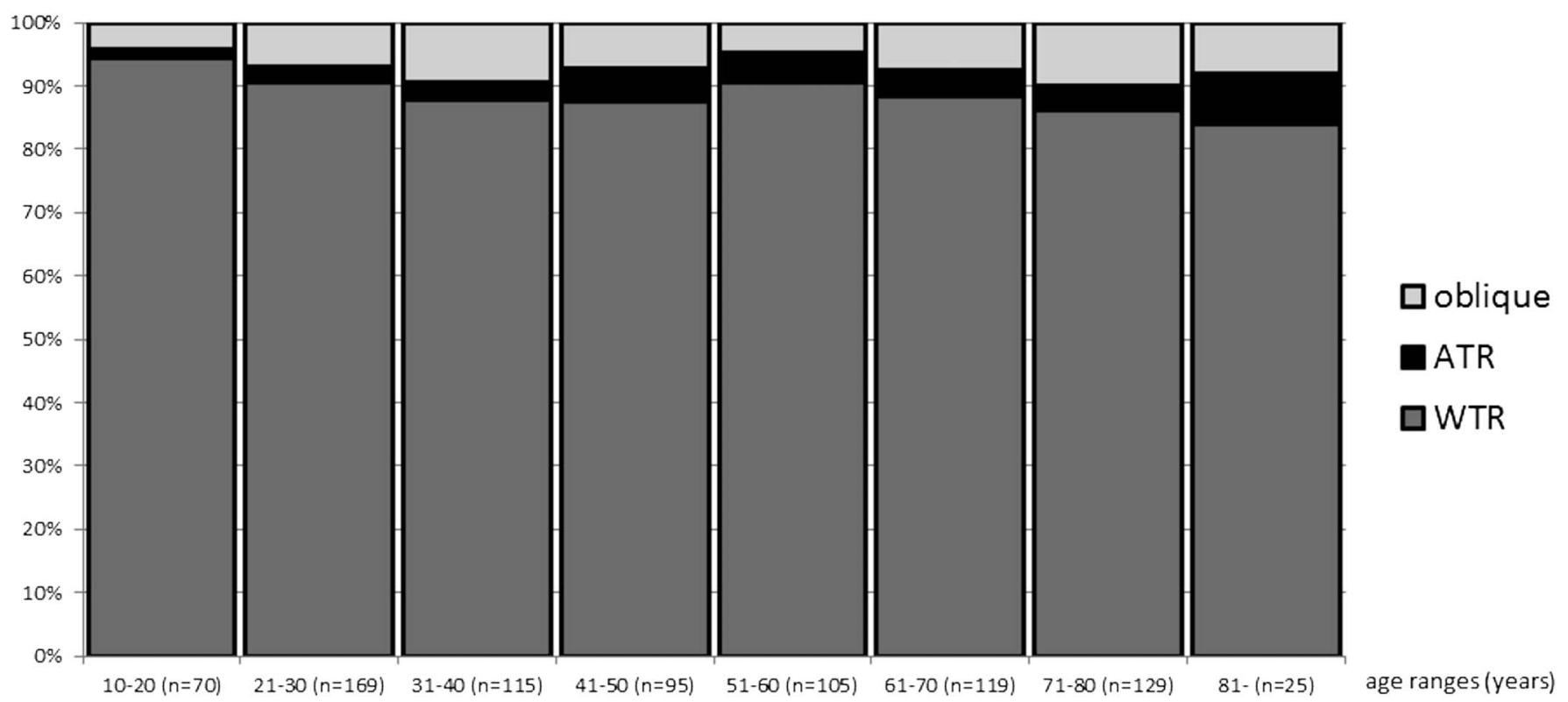

FIGURE 2. Age dependence of the orientation of the posterior corneal astigmatism. The $x$ axis denotes the age groups in years, and the $y$ axis denotes the prevalence of types of astigmatism. 
Koch et $\mathrm{al}^{15}$ measured $-0.3 \mathrm{D}$ for the mean magnitude of posterior corneal astigmatism over the 1.0- to 4.0-mm zone with a dual Scheimpflug analyzer using ray tracing, which exceeded $0.50 \mathrm{D}$ in $9.0 \%$. In our patient group, we observed that posterior corneal astigmatism exceeded $0.5 \mathrm{D}$ in $12.56 \%$ of patients. It is important to recognize that the repeatability and reliability of keratometry with the Pentacam HR are good, and that it provides high interoperator intraclass correlation coefficients in clinical practice. ${ }^{26-28}$

The age-related change regarding the anterior and posterior corneas may have importance in surgical procedures for astigmatism corrections. Dubbelman et $\mathrm{al}^{24}$ concluded that the radius and asphericity of the anterior corneal surface and the radius of the posterior corneal surface are not significantly age dependent at the $7.0-\mathrm{mm}$ measurement range of the cornea. Using a dual Scheimpflug analyzer, Koch et $\mathrm{al}^{15}$ described how the anterior corneal steep meridian tends to change to ATR with age; however, at the posterior surface, this change was not significant. In their study ${ }^{15}$ on the posterior cornea, the percentage of eyes with a steep meridian aligned horizontally increased from $0 \%$ in the $20 \mathrm{~s}$ to $7 \%$ in the $70 \mathrm{~s}$ and $10 \%$ in the $80 \mathrm{~s}$. In another study of Ho et $\mathrm{al}^{29}$ on the posterior cornea, measured within the $3.0-\mathrm{mm}$ central zone, the percentage of eyes with WTR astigmatism or with a steep meridian aligned horizontally increased from $0 \%$ in the 21 - to 30 -year age group to $9.1 \%$ in the 71 -year and above age group. We can strengthen this observation about the nearly age-stable posterior corneal surface with the Pentacam HR device. In contrast, the orientation of the anterior corneal astigmatism significantly changed with advancing age.

In conclusion, we demonstrated in a large series of patients examined for the posterior cornea that the astigmatism in the back surface is nearly independent of age, in contrast to that for the anterior surface. Besides this, we demonstrated that 1 in 8 eyes exceeded $0.5 \mathrm{D}$ of posterior astigmatism, which may have clinical importance. During different types of the correction of astigmatism-such as a toric IOL - we may have to calculate using this different age dependence of the axis and the magnitude of the astigmatism of the anterior and posterior corneal surfaces.

\section{REFERENCES}

1. Abrahamsson M, Fabian G, Sjöstrand J. Changes in astigmatism between the ages of 1 and 4 years: a longitudinal study. Br J Ophthalmol. 1988; 72:145-149.

2. Dobson V, Fulton AB, Sebris SL. Cycloplegic refractions of infants and young children: the axis of astigmatism. Invest Ophthalmol Vis Sci. 1984; 25:83-87.

3. Fan DS, Rao SK, Cheung EY, et al. Astigmatism in Chinese preschool children: prevalence, change, and effect on refractive development. $\mathrm{BrJ}$ Ophthalmol. 2004;88:938-941.

4. Khan MI, Muhtaseb M. Prevalence of corneal astigmatism in patients having routine cataract surgery at a teaching hospital in the United Kingdom. J Cataract Refract Surg. 2011;37:1751-1755.

5. Hoffer KJ. Biometry of 7,500 cataractous eyes. Am J Ophthalmol. 1980; 90:360-368

6. Ferrer-Blasco T, Montés-Micó R, Peixoto-de-Matos SC, et al. Prevalence of corneal astigmatism before cataract surgery. J Cataract Refract Surg. 2009;35:70-75
7. Hoffmann PC, Hütz WW. Analysis of biometry and prevalence data for corneal astigmatism in 23,239 eyes. J Cataract Refract Surg. 2010;36: 1479-1485.

8. Ninn-Pedersen K. Relationships between preoperative astigmatism and corneal optical power, axial length, intraocular pressure, gender, and patient age. J Refract Surg. 1996;12:472-482.

9. Nemeth G, Szalai E, Berta A, et al. Astigmatism prevalence and biometric analysis in normal population. Eur J Ophthalmol. 2013; 23:779-783.

10. Turuwhenua J. Reconstructing ocular surfaces by Purkinje images: an exact ray approach. Ophthalmic Physiol Opt. 2009;29:80-91.

11. Tomidokoro A, Oshika T, Amano S, et al. Changes in anterior and posterior corneal curvatures in keratoconus. Ophthalmology. 2000;107: 1328-1332.

12. Schlegel Z, Hoang-Xuan T, Gatinel D. Comparison of and correlation between anterior and posterior corneal elevation maps in normal eyes and keratoconus-suspect eyes. J Cataract Refract Surg. 2008;34:789-795.

13. Piñero DP, Alió JL, Alesón A, et al. Corneal volume, pachymetry, and correlation of anterior and posterior corneal shape in subclinical and different stages of clinical keratoconus. J Cataract Refract Surg. 2010; 36:814-825

14. Cheng LS, Tsai CY, Tsai RJ, et al. Estimation accuracy of surgically induced astigmatism on the cornea when neglecting the posterior corneal surface measurement. Acta Ophthalmol. 2011;89:417-422.

15. Koch DD, Ali SF, Weikert MP, et al. Contribution of posterior corneal astigmatism to total corneal astigmatism. J Cataract Refract Surg. 2012; 38:2080-2087.

16. Bregnhøj JF, Næser K. Ocular residual astigmatism in the pseudophakic eye. Abstract from Congress of the European Society of Ophthalmology. 2013; EP-COR-230.

17. Koch DD, Jenkins RB, Weikert MP, et al. Correcting astigmatism with toric intraocular lenses: effect of posterior corneal astigmatism. J Cataract Refract Surg. 2013;39:1803-1809.

18. Edmund C. Posterior corneal curvature and its influence on corneal dioptric power. Acta Ophthalmol (Copenh). 1994;72:715-720.

19. Garner LF, Owens H, Yap MK, et al. Radius of curvature of the posterior surface of the cornea. Optom Vis Sci. 1997;74:496-498.

20. Módis L Jr, Langenbucher A, Seitz B. Evaluation of normal corneas using the scanning-slit topography/pachymetry system. Cornea. 2004; 23:689-694.

21. Dubbelman M, Sicam VA, Van der Heijde GL. The shape of the anterior and posterior surface of the aging human cornea. Vision Res. 2006;46: 993-1001.

22. Ho JD, Tsai CY, Tsai RJ, et al. Validity of the keratometric index: evaluation by the Pentacam rotating Scheimpflug camera. J Cataract Refract Surg. 2008;34:137-145.

23. Pinero DP, Saenz Gonzalez C, Alio JL. Intraobserver and interobserver repeatability of curvature and aberrometric measurements of the posterior corneal surface in normal eyes using Scheimpflug photography. J Cataract Refract Surg. 2009;35:113-120.

24. Dubbelman M, Weeber HA, van der Heijde RG, et al. Radius and asphericity of the posterior corneal surface determined by corrected Scheimpflug photography. Acta Ophthalmol Scand. 2002;80:379-383.

25. Montalbán R, Piñero DP, Javaloy J, et al. Scheimpflug photographybased clinical characterization of the correlation of the corneal shape between the anterior and posterior corneal surfaces in the normal human eye. J Cataract Refract Surg. 2012;38:1925-1933.

26. Crawford AZ, Patel DV, McGhee CN. Comparison and repeatability of keratometric and corneal power measurements obtained by Orbscan II, Pentacam, and Galilei corneal tomography systems. Am J Ophthalmol. 2013;156:53-60.

27. Chen D, Lam AK. Reliability and repeatability of the Pentacam on corneal curvatures. Clin Exp Optom. 2009;92:110-118.

28. Módis L Jr, Szalai E, Kolozsvári B, et al. Keratometry evaluations with the Pentacam high resolution in comparison with the automated keratometry and conventional corneal topography. Cornea. 2012;31:36-41.

29. Ho JD, Tsai CY, Liou SW. Accuracy of corneal astigmatism estimation by neglecting the posterior corneal surface measurement. Am J Ophthalmol. 2009; 147:788-795, 795.e1-e2 